\title{
Rechtsgeschichte
}

\section{Tilman Repgen}

\section{Produktive Unruhe - Kein Recht ohne Form}




\section{Produktive Unruhe - Kein Recht ohne Form*}

Der Band dokumentiert die Ergebnisse eines Kolloquiums der Gesellschaft für Reichskammergerichtsforschung zum Thema: »Formalismus und Formalitäten. Eine vergleichende Untersuchung des Kameralprozesses «. Seit Normdurchsetzung und Rechtspraxis größere Aufmerksamkeit finden, ist die Notwendigkeit, sich mit dem Zivilprozess $\mathrm{zu}$ befassen, deutlich gewachsen. Die Arbeiten von Peter Oestmann bedienen dieses Bedürfnis in prominenter Weise, stehen aber nicht allein. ${ }^{\mathbf{I}}$ In die Linie dieses Bemühens um ein besseres Verstehen des »alten " Prozesses gehört das hier angezeigte Werk. Trotz mancher Arbeiten in den letzten Jahrzehnten bleibt die Forschung noch immer auf die großen »Erzählungen « des I9. Jahrhunderts zum Prozess angewiesen. Von Bethmann-Hollwegs ${ }^{2}$ und Wetzells ${ }^{3}$ Darstellungen etwa sind bis heute nicht ersetzt. Auf einen systematischen Zugriff kann man aber auch bei Fallstudien etc. nicht verzichten, wenn man Orientierung im Gewirr der Einzelheiten sucht. Dennoch wird dieser Rückgriff auf die Werke des I9. Jahrhunderts immer problematischer, da eben auch in der Historiographie des Zivilprozesses Entwicklungen stattfinden. So wie manche Auffassungen über das I9. Jahrhundert im Bereich der Methode, der Privatrechtsprinzipien oder der biographischen Einschätzungen in den letzten Jahren dekonstruiert worden sind, steht auch die Geschichte des Zivilprozesses auf dem Prüfstand. Davon zeugt dieser Tagungsband, der manche »Einsichten « korrigiert. Die Beiträge kreisen um die Funktion von Formen im Gerichtsverfahren. Zugleich öffnete sich die Fragestellung auch für die Wirkungen der Formerfordernisse auf das Verhältnis der Bevölkerung zur Justiz sowie die Transaktionskosten der Jus- tiz, die freilich nicht ohne Berücksichtigung der Vollstreckungsmöglichkeiten, letztlich also der Möglichkeit der Verwirklichung von Rechtsansprüchen zu bestimmen sind.

Peter Oestmann leistet in seinem Beitrag »Die Zwillingsschwester der Freiheit. Die Form im Recht als Problem der Rechtsgeschichte « (I-54) eine fulminante Dekonstruktion der vor allem von Wilhelm Ebel geprägten Entwicklungsgeschichte des deutschen Rechts, die sich am Verhältnis zur Form ablesen lasse. Nach Ebel zeichnet sich eine archaische Epoche durch äußeren und inneren Formalismus aus, während entwickeltere Rechtsordnungen zur Formfreiheit tendierten und dem Willen des Individuums größere Bedeutung zollten. Die Ebel'sche Deutung steht in einem krassen Gegensatz zum Befund von Jhering, wonach die Form die »Zwillingsschwester der Freiheit « sei, mithin eine Begleiterscheinung einer Rechtsordnung, die der Freiheit großes Gewicht einräumt, wie es das I9. Jahrhundert tat. Jhering verband mit der Form Rechtssicherheit und Zurückdrängung von Willkür. Oestmanns Aufsatz liest sich wie eine Programmschrift. Tatsächlich sind die Querbezüge zu seinen sonstigen Arbeiten zahlreich. In Auseinandersetzung mit den maßgeblichen Arbeiten von Eberhard Schmidt, Ekkehard Kaufmann, Wilhelm Ebel sowie auch der sonstigen Literatur verwirft Oestmann auf der Grundlage eigenen Quellenstudiums die These einer signifikanten Veränderung des Verhältnisses der Rechtsordnung zur Form am Beginn der frühen Neuzeit. »Recht ohne Form gab und gibt es nicht " (52). An die Stelle einer leicht überschaubaren Skizze setzt Oestmann nun ein »Wimmelbild «. Es hat die Schwierigkeit, dass der Über-
Zwischen Formstrenge und Billigkeit. Forschungen zum vormodernen Zivilprozeß, hg. von Peter Oestmann (Quellen und Forschungen zur höchsten Gerichtsbarkeit im Alten Reich 56), Köln, Wien, Weimar: Böhlau 2009, xiii, 342 S., ISBN 978-3-4I 2-20324-5
I Vgl. etwa Wolfgang Sellert, Prozeßgrundsätze und Stilus $\mathrm{Cu}$ riae am Reichshofrat im Vergleich mit den gesetzlichen Grundlagen des reichskammergerichtlichen Verfahrens, Aalen I973; besonders hervorzuheben: BETTINA Dick, Die Entwicklung des Kameralprozesses nach den Ordnungen von I 495 bis I 555 , Köln, Wien I98I; aus neuerer Zeit etwa: STEFfen Schlinker, Litis Con- testatio. Eine Untersuchung über die Grundlagen des gelehrten $\mathrm{Zi}$ vilprozesses in der Zeit vom I 2. bis zum I9. Jahrhundert, Frankfurt am Main 2008.

2 Der Civilprozeß des gemeinen Rechts in geschichtlicher Entwicklung, Bonn I864-I874.

3 System des ordentlichen Civilprozesses, 3. Aufl. Leipzig I 878 . 
blick zunächst eine sorgfältige Vertiefung in die Quellen voraussetzt, aber es hat den unbestreitbaren geschichtswissenschaftlichen Vorteil, eben aus diesen zu schöpfen.

In Anlehnung an einen Aufsatztitel von Fritz Baur (1963) behandelt Joachim Münch »Richtermacht und Formalismus im Verfahrensrecht " (55-IO3). Gemeint ist das zivilrechtliche Erkenntnisverfahren. Münch geht es um die Balance von "Formalisierung « des Verfahrens auf der einen und richterlicher Entscheidungs- und Gestaltungsmacht auf der anderen Seite. Dieses Spannungsverhältnis wird mit einer dritten Größe konfrontiert: der ökonomischen Analyse. Die Ergebnisse der »Courts «-Studie der Weltbank weist Münch allerdings als untauglich zurück, um daraus Rückschlüsse auf die Tauglichkeit des Prozessrechts zu ziehen. Dies alles wird vorwiegend anhand einer normativen Betrachtungsweise des geltenden Rechts untersucht. Das Thema »Richtermacht « ruft freilich auch nach historischer Analyse.

Bernhard Diestelkamp korrigiert in einem völlig aus den Quellen gearbeiteten Aufsatz die »Beobachtungen zur Schriftlichkeit im Kameralprozeß « (IO5-II5) insofern, als er auf die bedeutende Rolle mündlicher Prozesshandlungen aufmerksam macht - spiegelbildlich zur Bedeutung der Schriftlichkeit im modernen »mündlichen « Zivilprozess. - Eva Ortlieb meint allerdings, beim Reichshofrat seien die mündlichen Elemente über das Maß beim Reichskammergericht hinausgegangen (»Das Prozeßverfahren in der Formierungsphase des Reichshofrats [I 5 I9I 564] «, I I 7-I 3 8). Insgesamt sieht Ortlieb beim Reichshofrat in der Anfangsphase weniger Förmlichkeit als beim Reichskammergericht. - Fraglos bietet die förmliche Streitbefestigung durch die litis contestatio einen besonders geeigneten $\mathrm{Ge}-$ genstand für das Rahmenthema. Steffen Schlin- ker, derzeit der beste Kenner der Materie, zeichnet das Eigenleben dieses antiken Relikts nach, das auch nach seiner Abschaffung im I7. Jahrhundert noch rechtssystematische Wirkung hatte (I39-I64). - Eher einen Hintersinn von »Formstrenge « betrifft die Abhandlung von Filippo Ranieri über »Entscheidungsfindung und Begründungstechnik im Kameralverfahren « (I65I90), in der es um die formalen Aufbauregeln der Relationen geht. Außerdem macht er auf die Präjudizialwirkung kammergerichtlicher Entscheidungen aufmerksam, die auf einer auch dem common law geläufigen Argumentationskultur induktiver Erfahrungssätze aufbaut - eine bislang kaum beachtete Gemeinsamkeit. - Barbara Stollberg-Rilinger erzählt von einem Sesselstreit im Reichskammergericht, der das Kollegium im Unterschied zum Reichshofrat als ein repräsentatives, gleiches und unabhängiges Organ erscheinen lässt - gar nicht das, was man im Ständestaat erwartet (I9I-2I6). - Steffen Wunderlich zeigt, dass das Reichskammergericht von Anfang an selbstbewusst auch gegenüber dem Kaiser auf der Einhaltung der Prozessformen bestand und damit, so ließe sich folgern, Unabhängigkeit entwickelte (2I7-246). - Ignacio Czeguhn lenkt den Blick auf den Zivilprozess in Spanien in der Neuzeit bis zur Reform von I 855 (247-265). - Alain Wijffels behandelt Frankreich im napoleonischen Zeitalter, das dem Richter eine aktive, gestaltende Rolle im Prozess zuerkannte (267-29I). - Hans-Peter Haferkamp spürt schließlich dem "gefühlten Fortwirken « des Kameralprozesses im I9. Jahrhundert nach und verwickelt einmal mehr die großen Namen der Zeit in ein aufschlussreiches Gespräch, an dessen Ende der Kameralprozess sehr lebendig blieb (293-3 I0).

Die Lektüre der von Ansatz und Gegenstand her keineswegs einheitlichen, aber durchweg ge- 
lungenen Beiträge des Sammelbandes regt an, die aufgeworfenen und weiterführenden Überlegungen fortzusetzen - das ist nicht wenig. Stören Tagungen und Sammelbände auch mitunter die »Ruhe des Forschers « (VII), so lohnt es gewiss, sich durch diesen beunruhigen zu lassen. Inhaltlich führt diese Beunruhigung weit über das bisherige Verständnis von Form und Formstrenge hinaus. Das alte Bild einer sich von archaischer Förmlichkeit zu liberaler Formfreiheit wandelnden Welt des Rechts ist überholt.

Tilman Repgen

\section{Westschweizerische Selbstbespiegelungen*}

Beide Bücher verbinden höchst äußerliche Anlässe: 2008 feierte die - als solche erst I 890 benannte - Universität Lausanne den 300 . Jahrestag der Errichtung des ersten, dauerhaften juristischen Lehrstuhls, und am I9. Oktober 2008 wählten die Genfer einen Verfassungsrat, der bis 2012 eine neue Kantonsverfassung ausarbeiten soll.

Als Gründungsjahr ihrer juristischen Fakultät betrachten die Lausanner das Jahr I708: Damals berief die Berner Obrigkeit Jean Barbeyrac, der allerdings erst I 7 I I kam und schon I 7 I 7 in die Niederlande weiterzog. Was nichts ändert, dass er als der erste und zugleich bis heute bekannteste Lausanner Rechtslehrer erscheint. Die rechtswissenschaftliche Fakultät hat sich zur Erinnerung an diesen Anfang eine Art Festschrift gegönnt, verfasst von ihren gegenwärtigen und früheren Mitgliedern und einem Bundesverwaltungsrichter. Entgegen dem Titel befasst sich der Band nicht ausschließlich damit, die 300jähige Geschichte des Rechtsunterrichts in der Waadtländer Hauptstadt darzustellen. Vielmehr werden, wie es eben für solche Publikationen üblich ist, Haupt- und Randthemen der Rechtswissenschaft abgehandelt: Das reicht von der Beweiskraft der DNA-Analyse über rück- wirkende Restrukturierungen im Fusionsgesetz bis zur Behandlung vorsorglicher Maßnahmen und der Rechtshilfe in ausländischen Scheidungsverfahren. $\mathrm{Zu}$ diesen und andern "modernen « Sachthemen gesellen sich persönlichere Beiträge: So macht sich der gegenwärtige Inhaber des Lehrstuhls für Deutsches Recht, Götz Schulze, über dessen Zukunft angesichts der BolognaReformen Gedanken (3OI-3II) oder sein VorVorgänger Fritz Sturm blickt nicht ohne ein gerüttelt Maß an Eitelkeit auf seine 40 Jahre Lehrtätigkeit zurück (313-335). Wenn, wesentlich bescheidener in ihrer Selbstdarstellung, Suzette Sandoz, die erste juristische Ordinaria, in ihrer für den Druck überarbeiteten Abschiedsvorlesung von 2006 unter anderem den Wert des Lateins verteidigt (289-300), ist das zwar objektiv richtig, aber - Bologna lässt grüßen - auch restlos überholt: Es gibt Dinge, die man ein für alle Mal erledigt hinnehmen muss. Was gleich auf Zeile 4 des Vorworts dem Lateinkundigen schmerzlich bewusst wird, schreiben doch die Herausgeber ungerührt vom »ius naturalis ", was nicht nur für sie, sondern nicht minder die sicher zahlreichen Mitleser und Korrektoren beschämend ist. Nicht besser der abwegige Fehler im Seitentitel zu Suzettes Sandoz' Aufsatz: Aus

\footnotetext{
" Denis Tappy, Bettina KahilWolfF, LÉONARd BRUChEZ, 300 ans d'enseignement du droit à Lausanne (Recherches juridiques lausannoises 46), Genf, Zürich, Basel: Schulthess 2010, XVI, 44I S., ISBN 978-3-7255-6109-4; James Fazy, De l'intelligence collective des sociétés. Cours de législation constitutionnelle, hg. von Michel Hottelier, Genf, Zürich, Basel: Schulthess 2010,
}

XXXIX, 448 S., ISBN 978-3-725 5-6067-7 